\title{
CONTROLLERS FOR FAULT TOLERANT ACTUATORS
}

\section{B. Dołęga, P. Rzucidło}

Rzeszów University of Technology, Department of Avionics and Control, ul. W. Pola 2, 35-959 Rzeszów, Poland.

E-mail:dolbog@prz.edu.pl,pawelrz@prz.edu.pl

Received 2006-10-16, accepted 2007-03-20
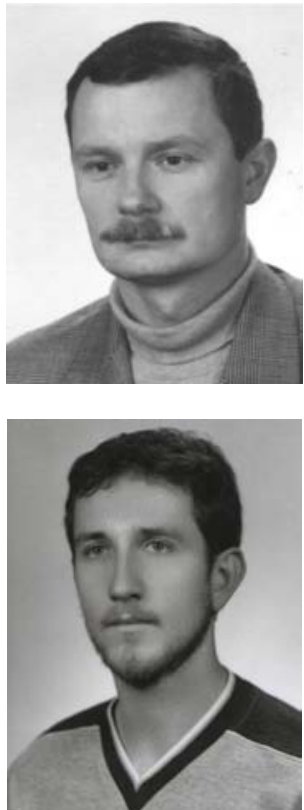

Bogusław DOŁĘGA, PhD. Eng

Education: Rzeszow University of Technology.

Affiliations and functions: Rzeszow University of Technology, assistant; 1994 - PhD degree from the Department of Mechanical Engineering of Rzeszow University of Technology; 1995 to present — Assistant to Professor on Avionics and Control Systems Department at the University.

Research interests: Diagnostic aircraft navigation and control systems, with a focus on the development of the control theory for online fault detection and localization methods.

Experience: Co-organizer of Avionics Conferences.

Publications: Over 40 scientific articles.

Present position: Assistant to Professor on Avionics and Control Systems Department at the University.

\section{Paweł RZUCIDŁO, PhD. Eng.}

Education: M.Sc. (Eng.) degree in Aeronautics, Rzeszów University of Technology, Faculty of Mechanical Engineering and Aeronautics. 2001 to 2005: PhD. studies, Rzeszów University of Technology, Faculty of Mechanical Engineering and Aeronautics, Specialization: Machine Building and Exploitation.

Research interest: Aircraft Control with an Emphasis on Interactions Between Human and Machine.

Publications: 19 scientific articles and conference papers.

Present position: 2005 to present: Researcher, Rzeszów University of Technology, Department of Avionics and Control.

Abstract. The Fly-by-Wire technology enables flexible shaping of both effective dynamics and handling qualities of aircraft. This solution extends aircraft possibilities and supports the pilot by use of high-level control laws. Application of FBW for aircraft would make flying both safer, and more popular. On the other hand the FBW system must be highly reliable. Advanced indirect flight control technology requires the use of actuators characterized by fault tolerant architecture. It should enable improvement of reliability of the aircraft control system and safety of performed flights effectively. The Electromechanical Actuator (EMA) consists of the electric motor, gearbox and controller. The actuator controller should be made intrinsically reliable and should be insensitive to other equipment failure. The conception of fault tolerant control algorithms as well as practical realization of it has been presented in this work. Tests of reliability of the complete EMA unit have also been presented herein.

Keywords: actuator, controller, Power-by-Wire, Fly-by-Wire.

\section{Introduction}

Fly-by-wire (FBW) systems have been applied since the end of the 1960s. In the beginning they were analogy solutions. They allowed aircraft to overcome problems with conventional systems (for example Concorde), or they were experimental installations [11]. The first digital FBW was used on the F-8 Crusader aircraft and then in the space shuttle program, where it was a sheer necessity as task performance would have been impossible with conventional mechanically augmented configurations. This technique allows an extension of possibilities for aircraft control, stabilization of flight parameters and enables better flexible automatic control. Indirect control of aircraft improves flight safety and minimizes pilot load. The development of digital technology makes FBW systems more profitable and the next step seems to be using these systems in general aviation aircrafts $[3,12$, 4]. Indirect control of aircraft improves flight safety and minimizes pilot load. The pilot can work as computer operator and also control the plane classically by using the control wheel or side stick in standard mode [6]. For this kind of control, actuators are responsible for movements of aircraft control surfaces. For these aircrafts in particular, actuators are critical elements of the FBW control systems. 


\section{The conception of fault tolerant actuators}

The electromechanical actuator (EMA) realized in power-by-wire (PBW) technology seems to be the next major step in the development of aircraft control. In this solution, control power comes directly from the aircraft's electrical system. Removing hydraulic systems would greatly reduce the amount of support equipment and maintenance costs [5]. On the other hand actuators are critical elements of the FBW system and they should perform strict criteria. They have to be reliable and work stably in every situation. It is very important because an actuator failure can cause serious accidents. The control surface actuator should be precise, fast, powerful and reliable [2]. An important part of this element is the electronic controller. Reliability of the EMA can be improved by using the appropriate hardware structure of micro controller, and also by using adequate control techniques and software solutions.
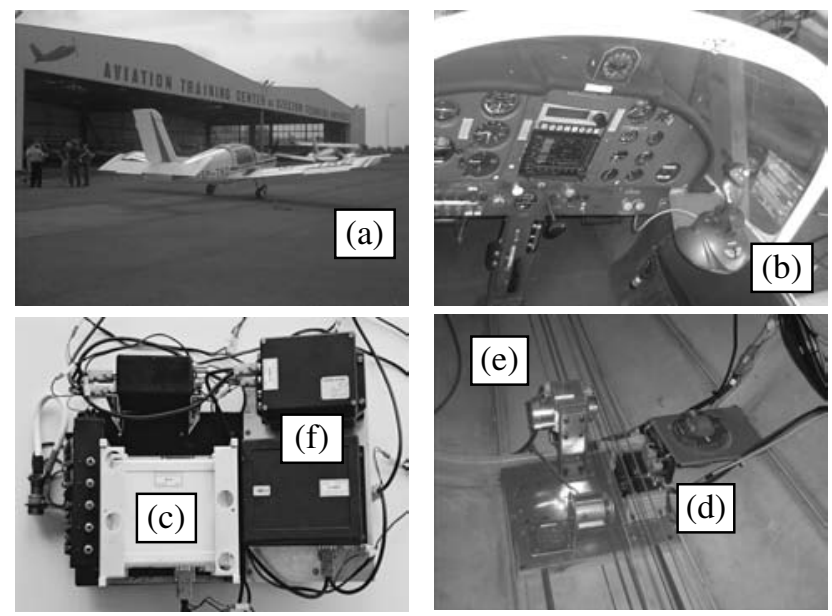

Fig 1. General view of PZL-110 "Koliber" aircraft (a), cockpit (b), main parts of SPS-1 system: flight control computer (c), controller (d), electromechanical unit (e), measurement equipment (f)

\section{Data transmission network}

The actuator should be intrinsically reliable and insensitive to other equipment failures. This second feature can be achieved by multiplication of superior control circuits: data buses, flight computers and measurement equipment. The structure and pictures of the SPS-1 indirect flight control system are presented in figures 1-2. This system has been designed at the Department of Avionics and Control, Rzeszów University of Technology. The data transmission network of SPS-1 is based on the serial communication bus CAN2.0 [9]. The CAN standard was developed in the 1980s for the automotive industry [1]. Since then, this standard has been disseminated and is used in many fields of technology. It defines hardware and data transmission methods as well as ways of solving conflicts and bus failure detection. Units and tools necessary for its service are easily available and this standard is characterized by good performance. The probability of data failure in a CAN network is around $10^{-13}$ per message transmission [1]. Assuming the full load of the bus (around 8000 messages per second at high-speed $1000 \mathrm{Mb} / \mathrm{s}$ ), this will result in a probability of $2.9 * 10^{-6}$ undetected failures per flight hour [10].

The SPS-1 system CAN data buses are doubled and three flight control computers (FCCs) are connected to both. Redundant system architecture is supported by the CAN aerospace data transmission protocol [10]. This standard requires 29-bit identifiers of data frames. In order to create redundant identifiers, a constant value must be added. In CAN aerospace v1.6, the value equalled 10000 (decimal). Established CAN standard of data transmission is very reliable and fault tolerant. It also enables the performance of advanced real-time laboratory and in-flight tests of the controller. Implementation of the original CAN aerospace protocol was difficult because of the decimal value added to redundant controller (and other device) identifiers [10]. The main problems were filtering and masking of identifiers by micro controllers. Every CAN controller has a limited number of accessible identifier mask registers and practical realization of the receipt of data was very hard and even impossible in many cases. Our team proposed that the authors of CAN aerospace change the offset value to a hexadecimal value and this suggestion was accepted. The next version of protocol will be changed in this point.

\section{Structure of EMA controller}

The EMA controller is connected directly to the electrical system of the aircraft and the electrical engine is powered through a built-in power output (Fig 3). The control signals are delivered to a micro controller from three independent flight control computers (through CAN buses) and one extra line is connected directly to the sidestick (emergency control). The controller hardware is based on a microprocessor equipped with a watchdog timer, an A/D converter, three I/O ports, and a capture compare module, which is used for catching PWMs (pulse width modulation signals). The microprocessor is galvanically isolated from input and output circuits by transports. The power is supplied from a DC/DC converter. A security chip powered from the board power lines of the aircraft assures it. Feedback from deflection is realized by a potentiometer installed in the electromechanical unit and is powered by the controller circuit.

The software of the EMA controller is realized in an assembler. Low-level programming allows avoidance of possible high-level compiler errors and makes softwarehardware relations clearer. Particular procedures and functions of the software were optimized and tested separately. During successive stages of software development, interconnections were also tested. The complete software was implemented on hardware and tested under CAN Monitor application supervision [9]. 


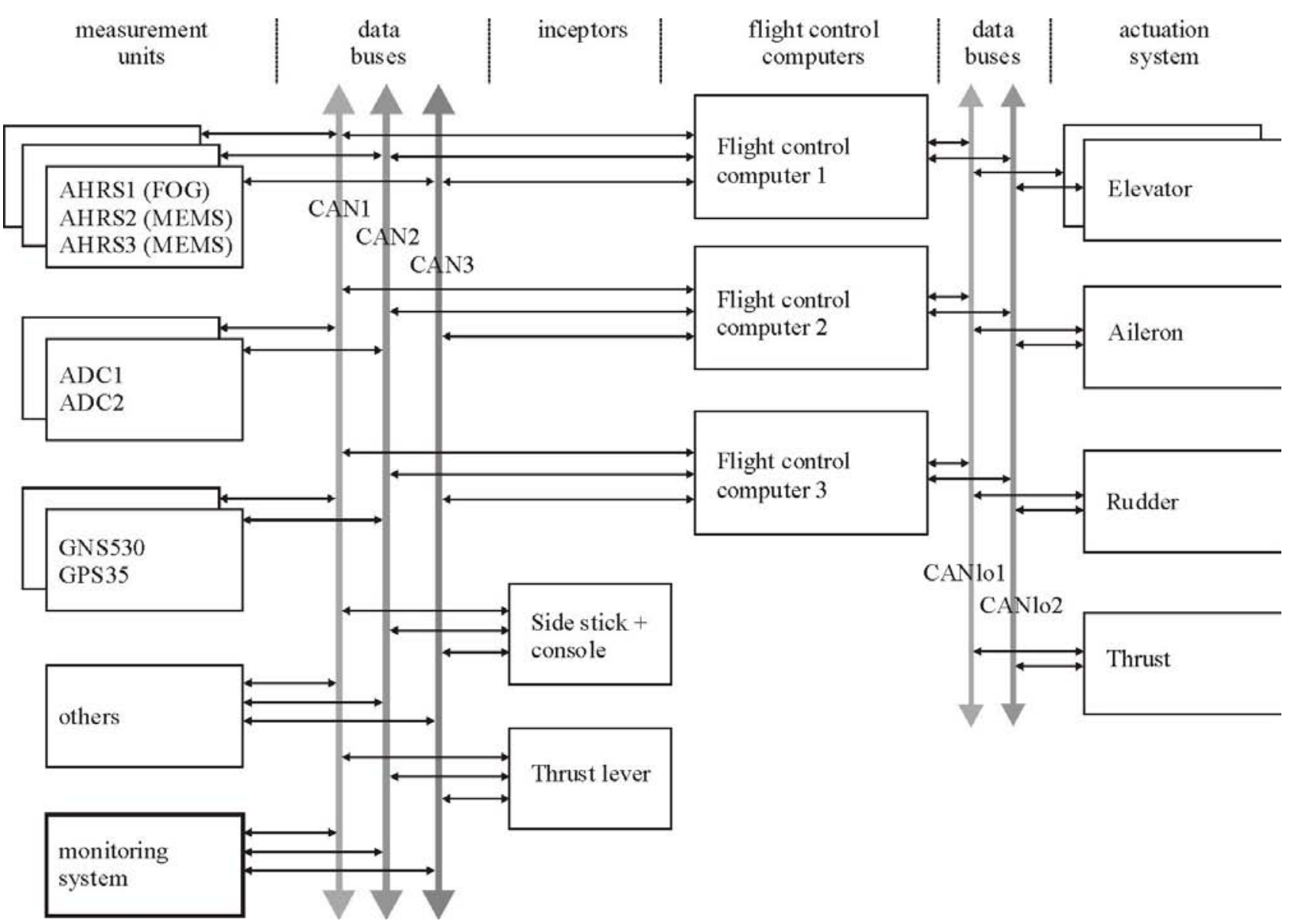

Fig 2. Block scheme of SPS-1 system and set of electromechanical actuators

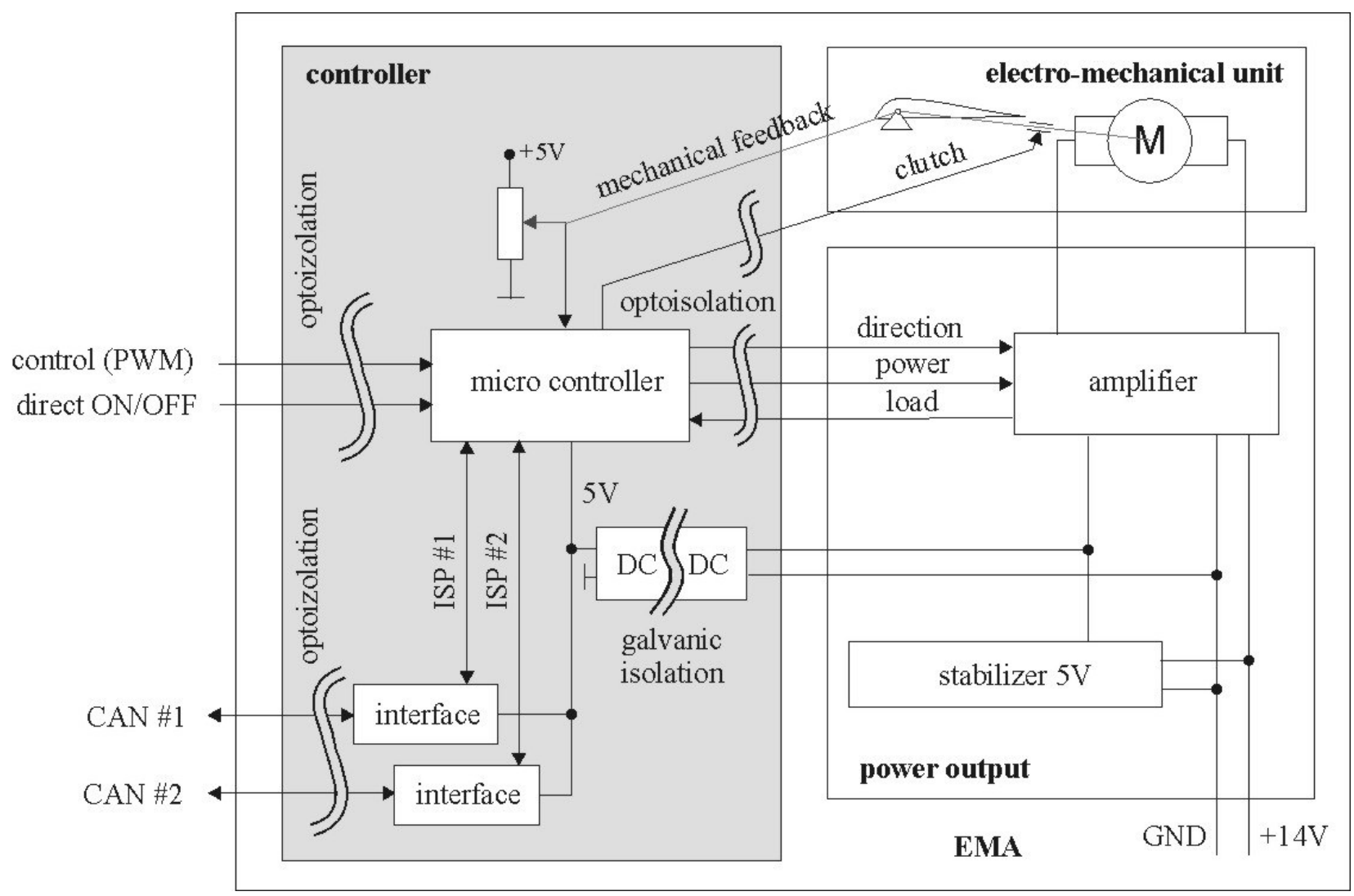

Fig 3. Scheme of EMA controller 


\section{Control algorithms}

The actuator controller receives data from three independent flight control computers through two CAN data buses. From all the received signals only one control signal is selected and it is only used for positioning the control surface. The selection process is repeated equally in every cycle of controller work 20 times per second. Two fault tolerant low-speed CAN data buses are used to improve the reliability of the transmission network and, in effect, one control signal out of three received is chosen (not one from six). Doubled data frames are sent from the same flight control computer (FCC) through different lines and they refresh the same controller register. The latest one is used during the next computations.

\section{Selection of control signals}

The selection process consists of these steps. Improper values (too low, too high or unexpected) are rejected at first. Desired positions of control surfaces (received from FCCs) are verified and sorted according to the highest value. If the differences between neighbour signals are correct, a medium value signal is chosen as the desired position of the control surface. The selection algorithm generates warning and error diagnostic frames to all FCCs in case of improper values of transmitted data or in case of high differences between the received signals. Information about outdated or not received data fames is also sent to all computers. At least two verified, up to date and low differenced signals are required for the selection process. Particular cases of selection algorithms are presented in table.

Table. Particular cases of selection algorithm

\begin{tabular}{cccccc}
\hline S1 & S2 & S3 & $\Delta_{1}$ & $\Delta_{2}$ & Voted \\
\hline$\bullet$ & $\bullet$ & $\bullet$ & $\sqrt{ }$ & $\sqrt{ }$ & S2 \\
$\bullet$ & $\bullet$ & $\bullet$ & $\sqrt{ }$ & $\mathrm{x}$ & S2 \\
$\bullet$ & $\bullet$ & $\bullet$ & $\mathrm{x}$ & $\sqrt{ }$ & S2 \\
$\bullet$ & $\bullet$ & $\bullet$ & $\mathrm{x}$ & $\mathrm{x}$ & $\mathrm{st}$ \\
$\bullet$ & $\bullet$ & $\circ$ & $\sqrt{ }$ & - & $\mathrm{S} 2$ \\
$\bullet$ & $\bullet$ & $\circ$ & $\mathrm{x}$ & - & $\mathrm{st}$ \\
$\bullet$ & $\circ$ & $\circ$ & - & - & $\mathrm{st}$ \\
$\circ$ & $\circ$ & $\circ$ & - & - & $\mathrm{st}$ \\
\hline
\end{tabular}

S1-3 - received and sorted according to the highest value signals from FCCs,

$\circ / \bullet \quad$ - absence / presence of signal,

$\sqrt{ } / \mathrm{x}$ - acceptable / non acceptable difference,

st - desired position of control surface delivered directly from pilot inceptor.

If selection between signals delivered from FCCs is impossible, all control surfaces are frozen in their last position and a pilot can deflect the controls be Ed via his side-stick (or control-wheel). The pilot can switch control into stick input at any moment during the flight by switching DIRECT to ON position. To achieve high reliability this process is done without the use of FCCs. The control signals are transmitted directly from the sidestick to the actuator controllers through PWM (pulse width modulation) lines. The assumed standard of PWM enables the detection of a short circuit or broken external circuits. To achieve this, a periodical signal with two constant zones, "0" and " 1 " have been adopted. Filling the PWM input is considered as a short circuit, and a steady zero, as a break. An example of time domain analysis of voting algorithm obtained during real-time tests of the controller is presented in figures 4-6 [5].

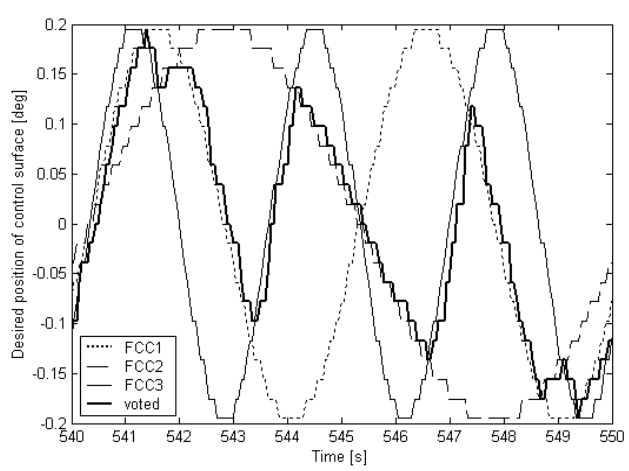

Fig 4. Test 1 of selection algorithm: differences between signals received from all FCCs are in acceptable range

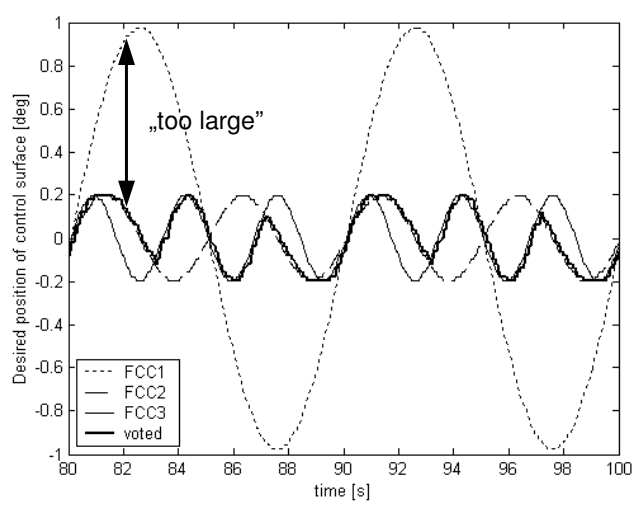

Fig 5. Test 2 of selection algorithm: signal from $F C C 1$ is " $t o o$ high" in some periods, difference between $F C C 2$ and $F C C 3$ is acceptable - device working correctly

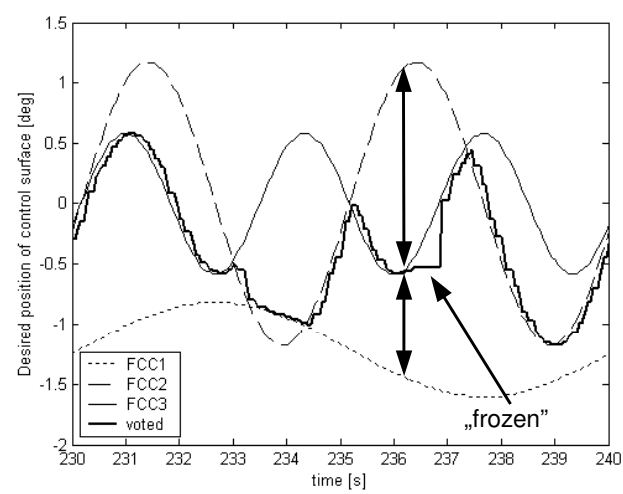

Fig 6. Test 3 of selection algorithm: both differences between signals received from FCCs are out of range in one period of time, control is "frozen" in this case 


\section{Heuristic regulator}

A variable and heavy load of the actuator or frequent changes of the control signal (especially direction) can cause quick amortization of the electrical motor and the power driver. The control algorithm should prevent situations where the motor will be overloaded for a long time. After some test runs, the heuristic regulator has been adopted in the presented solution. It uses dependences between motor powering and actuating error established by an expert. The heuristic regulator has very good static properties and good dynamic properties under rate saturation. It also allows minimization of the disadvantages of the wind-up effect to a high degree. Practical realization of this algorithm is very simple, the dependence between motor powering and actuating error can be formulated as (1)-(3):

$$
\begin{gathered}
y_{h}(k+1)=y_{h_{-} p}(k+1)+y_{h_{-} i}(k+1) \\
y_{h_{-} p}(k+1)=k p(e(k)) \\
y_{h_{-} i}(k+1)=\left\{\begin{array}{ccc}
y_{h_{-} i_{-} \max }(e(k)) & \text { if } & y_{h_{-} i}(k)+k i(e(k)) \cdot e(k) \cdot T_{d}>y_{h_{-} i_{-} \max }(e(k)) \\
y_{h_{-} i_{-} \min }(e(k)) & \text { if } & y_{h_{-} i}(k)+k i(e(k)) \cdot e(k) \cdot T_{d}<y_{h_{-} i_{-} \min }(e(k)) \\
\text { otherwise } & y_{h_{-} i}(k)+k i(e(k)) \cdot e(k) \cdot T_{d}
\end{array}\right.
\end{gathered}
$$

$\mathrm{T}_{\mathrm{d}} \quad$ - sample time,

$\mathrm{k}$ - period of time,

$\mathrm{y}_{\mathrm{h}}(\mathrm{k}) \quad$ - output signal of heuristic regulator,

$\mathrm{y}_{\mathrm{h}_{\mathrm{p}} \mathrm{p}}(\mathrm{k}) \quad$ - proportional component of output signal,

$\mathrm{y}_{\mathrm{h}_{-} \mathrm{i}}(\mathrm{k}) \quad$ - integral component of output signal,

e (k) - actuating error,

$\mathrm{kp}(\mathrm{e}(\mathrm{k})) \quad$ - expert chosen thresholds for gain in proportional path,

ki (e (k)) - expert chosen thresholds for gain in integral path,

$\mathrm{y}_{\mathrm{h} \_\mathrm{i} \_ \text {max }}(\mathrm{e}(\mathrm{k}))$ - maximal value of integral component of output signal (dependent on actuation error).

\section{Conclusion}

The controller operation has been checked in many possible situations. Positive results from laboratory tests made it possible to install a complete set of servos and controllers onboard the PZL-110 "Koliber" aircraft, as components of the experimental SPS-1 system. To achieve a higher safety level in the SPS-1 system, a redundant elevator servo and controller system has been adopted. A total of 12 flight hours with inputs and responses in various flight conditions have been recorded. Tests performed in flight (July-November 2003) supported the laboratory results. The next run of advanced in-flight tests of actuators is planned for summer 2006.

\section{References}

1. CAN Specification Version 2.0, Robert Bosch GmbH (1991).

2. DOŁEGA, B.; RZUCIDŁO, P. The conception of fault tolerant actuators (in Polish). In VII KKN-T The Diagnostics of Industrial Processes Conference. Rajgród, 2005, p. 273-275.

3. GRUSZECKI, J.; GRZYBOWSKI, J.; RZUCIDŁO, P. Electro-mechanical actuators for general aviation fly-by-wire aircraft. Aviation. Vilnius: Technika, 2005, vol. IX, no 1, p.19-25.

4. Integrated control system for general aviation aircraft (in Polish). In Polish State committee for scientific research. Ed by A. Tomczyk. Rzeszów, 2003, 8 T12C 04920.
5. JENSEN, SC.; JENNEY, GD.; DAWSON, D. Flight test experience with an electromechanical actuator on the F-18 systems research aircraft. In Nasa Report: NASA-TM-2000-209015, 2000.

6. ROGALSKI, T. The conception of the pilot friendly control system for small local communication aircraft. In AIAA 2002-4871, Monterey, California, 2002.

7. ROGALSKI, T.; DOŁEGA, B. The laboratory stand intends to test and prototype control system for small transportation aircraft. In International Multidisciplinary Conference. Baja Mare, 2003.

8. RZUCIDŁO, P. Monitoring and data acquisition system for experimental general aviation fly-bywire aircraft. Aviation. Vilnius: Technika, 2006, vol. X, no. 3, p. 16-20.

9. RZUCIDŁO, P. Monitor of CAN bus applied in the fly-by-wire control system of PZL-110 aircraft (in Polish). Diagnostyka PTDT. 2004, vol. 2, no. 32 .

10. STOCK, M. CAN aerospace interface specification for airborne CAN applications V 1.6, Revision 1.6, 12.01.2004, URL. In www.stockflightsystems.com

11. TOMCZYK, A. Digital flights control systems. Rzeszów: OWPRz, 1999.

12. TOMCZYK, A. Experimental fly-by-wire control system for general aviation aircraft. In AIAA2003-5776, AIAA Guidance. Navigation and Control Conference and Exhibit. Austin, Texas, 2003. 